\title{
Establishing The GZK Cutoff With Ultra High Energy Tau Neutrinos
}

\author{
A. Letessier-Selvon ${ }^{1}$ \\ Laboratoire de Physique Nucléaire et des Hautes Énergies, \\ IN2P3-CNRS, Universités Paris 6 \& 7, \\ 4 place Jussieu, Tour 33 RdC, 75252 Paris Cedex 05 France \\ e-mail : Antoine.Letessier-Selvon@in2p3.fr
}

\begin{abstract}
The cosmic ray spectrum has been shown to extend well beyond $10^{20} \mathrm{eV}$. With nearly 20 events observed in the last 40 years, it is now established that particles are accelerated or produced in the universe with energy near $10^{21} \mathrm{eV}$. In all production models neutrinos and photons are part of the cosmic ray flux. In acceleration models (bottom-up models), they are produced as secondaries of the possible interactions of the accelerated charged particle, in direct production models (top-down models) they are a dominant fraction of the decay chain. In addition, hadrons above the GZK threshold energy will also produce, along their path in the Universe, neutrinos and photons as secondaries of the pion photo-production processes. Therefore, photons and in particular neutrinos, are very distinctive signatures of the nature and distribution of the potential sources of ultra high energy cosmic rays. In the following we expose the identification capabilities of the Auger observatories. In the hypothesis of $\nu_{\mu} \rightarrow \nu_{\tau}$ oscillations with full mixing, special emphasis is placed on the observation of tau neutrinos, with which Auger is able to establish the GZK cutoff as well as to provide a strong and model independant constraint on the top-down sources of ultra high energy cosmic rays.
\end{abstract}

\section{INTRODUCTION}

The cosmic ray spectrum is now proved $[1,2]$ to extend beyond $10^{20} \mathrm{eV}$. To be observed on Earth with such energies, particles must be produced or accelerated in the Universe with energy near or above $10^{21} \mathrm{eV}$. Conventional acceleration mechanisms in astrophysical objects can only reach this requirement by stretching to the limit their available parameter space, making such scenarios unlikely to explain the origin of UHECR. Alternative hypotheses involving collapse of Topological Defects

1) Presented at the International Workshop on Observing Ultra High Energy Cosmic Rays From Space and Earth. August 9-12, 2000, Puebla (Mexico). 
(TD) or decay of Super Massive Relic Particles (SMRP) are well suited to produce particles above $10^{20} \mathrm{eV}$ but need a proof of existence.

Transport, from the source to Earth, is also an issue. At those extreme energies the Cosmic Microwave Background Radiation makes the Universe essentially opaque to protons, nuclei and photons which suffer energy losses from pion photo production, photo-disintegration or pair production. These processes led Greisen, Zatsepin and Kuzmin [3] to predict a spectral cutoff in the cosmic ray spectrum around $5 \times 10^{19} \mathrm{eV}$, the GZK cutoff. The available data although still very scarce do not support the existence of such a cutoff. Therefore the sources are either close by and locally over dense for the cutoff not to show, or some new physics prevent the UHECR from the expected energy losses against the CMB photons.

The following will briefly develop the arguments mentioned in this introduction. Interested readers should consult the numerous reviews devoted to the subject [4-6] for more details.

\section{OBSERVATIONS}

The differential spectrum of cosmic ray flux [7] as a function of energy is shown on Figure 1. Integrated fluxes above three energy values are indicated: 1 particle $/ \mathrm{m}^{2}$ second above $1 \mathrm{TeV}, 1$ particle $/ \mathrm{m}^{2}$-year above $10 \mathrm{PeV}, 1$ particle $/ \mathrm{km}^{2}$-year above $10 \mathrm{EeV}$. The energy spectrum is surprisingly regular in shape. From the GeV energies to the GZK cutoff, it can be represented simply by three power-law segments interrupted by two breaks, the so-called "knee" and "ankle".

Figure 2 is a zoom on the highest energy part of the total spectrum where only the latest AGASA data [2] is displayed. On this figure, the energy spectrum is multiplied by $E^{3}$ so that the part below the EeV energies becomes flat. Comparing the data points and the dashed line one has a clear view of what can be expected from a cosmological (uniform) distribution of conventional sources and what is observed.

The cutoff, that would be expected if the sources were cosmologically and uniformly distributed and if the observed cosmic rays had no exotic propagation or interaction properties, is not present in the observed data.

In the search for potential sources, one looks for correlations of the UHECR arrival directions with the distribution of matter within a few tens of Mpc. Such an analysis was done by the AGASA experiment for the highest energy range [8]. No convincing deviation from isotropy was found.

If the sources of UHECR are nearby astrophysical objects and if, as expected, they are in small numbers, a selection of the events with the largest magnetic rigidity would combine into multiplets (cluster of events whose error boxes overlap).

Figure 3 shows the subsample of events in the AGASA catalog with energies in excess of $100 \mathrm{EeV}$ (squares) and in the range 40-100 EeV (circles). One can see that there are three doublets and one triplet. The chance probability of having 


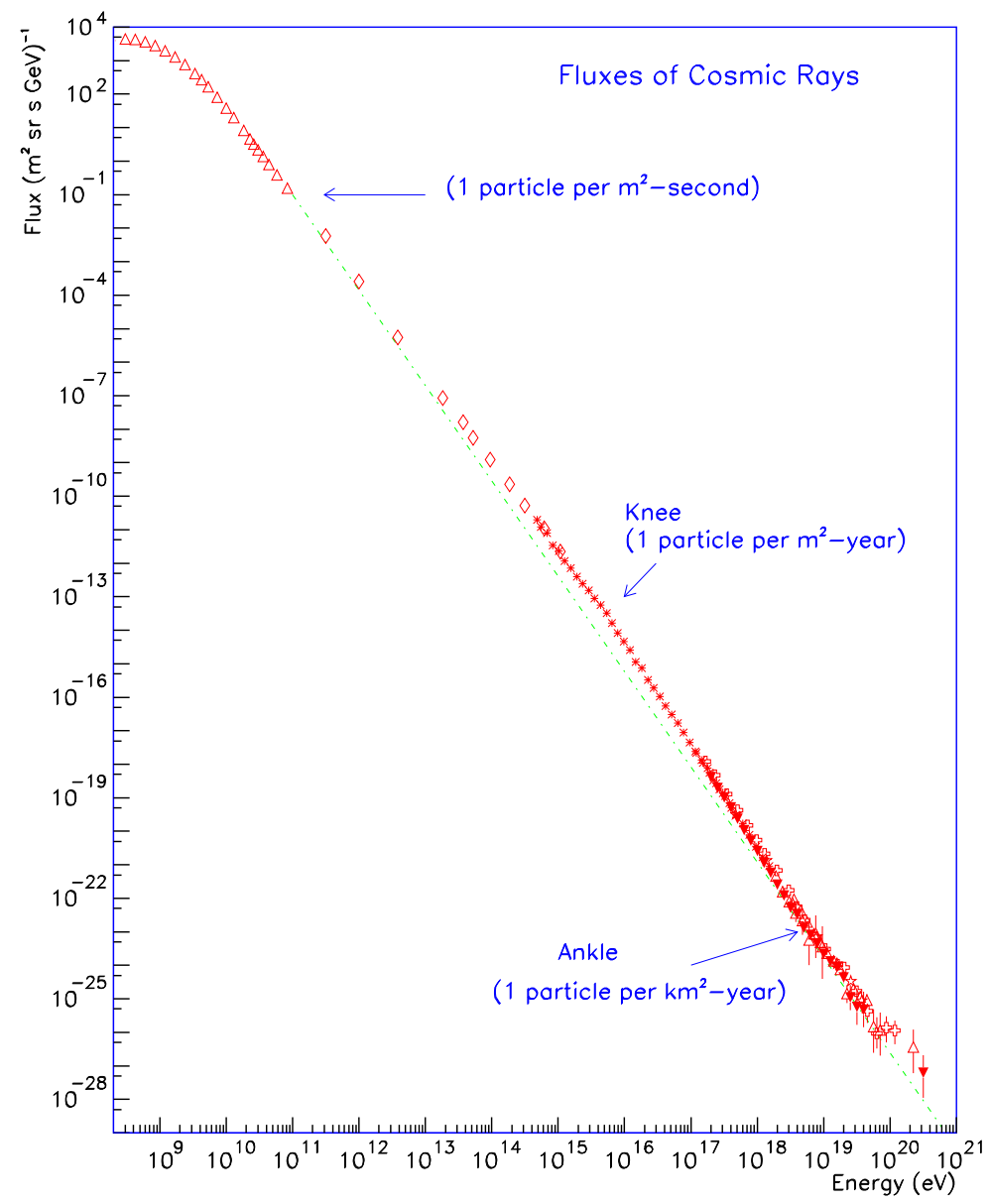

FIGURE 1. The cosmic rays spectrum

as many multiplets as observed with a uniform distribution is estimated to be less than $1 \%$

The non uniform sky coverage -all present detectors are in the northern hemisphere- and the small statistics available make anisotropy studies difficult. The Auger observatories are designed with full sky coverage and large detection areas to overcome these difficulties.

\section{TRANSPORT AND PRODUCTION}

Today's understanding of the phenomena responsible for the production of UHECR is still limited. One distinguishes two classes of processes: the "TopDown" and "Bottom-Up" scenarios. In Top-Down scenarios, the cosmic ray is a decay products of a super-massive particle. Such particles with masses exceeding $10^{21} \mathrm{eV}$ are either meta-stable relics of some primordial field or GUT gauge bosons 


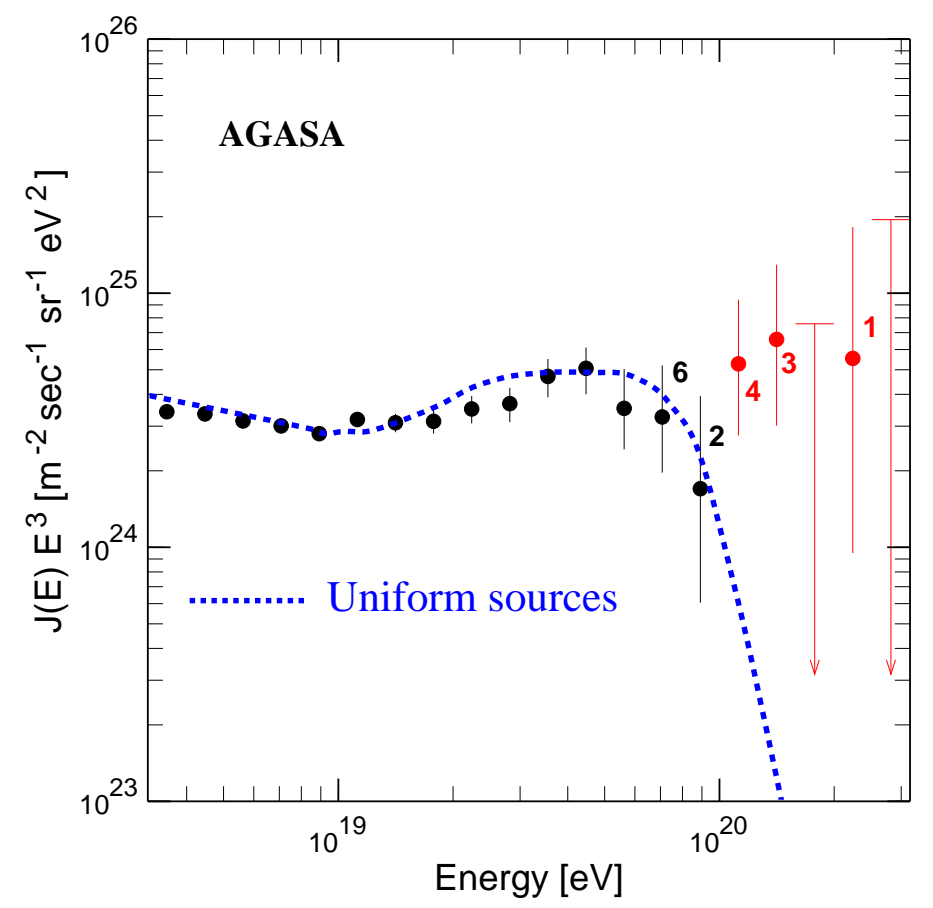

FIGURE 2. Highest energy region of the cosmic ray spectrum as observed by the AGASA detector. The figures near the data points indicate the number of events in the corresponding energy bin. The arrows show $90 \%$ confidence level upper limits. The dashed line is the expected spectrum if the sources were cosmologically distributed.

produced by the radiation or collapse of topological defects. In the Bottom-Up scenarios, the energy is transferred to a charged particle at rest through its electromagnetic interactions. This classical approach does not require new physics.

At energies above $10 \mathrm{EeV}$ and except for neutrinos, the Universe is not transparent to ordinary stable particles on scales larger than about $100 \mathrm{Mpc}$. Regardless of their nature, cosmic rays lose energy in their interaction with the various photon backgrounds, dominantly the copious Cosmic Microwave Background (CMB) but also Infra-Red and Radio. The absence of prominent visible astrophysical objects in the direction of the observed highest energy cosmic rays together with this distance limitation adds severe constraints on the "classical" Bottom-Up picture.

\section{A The GZK cutoff}

The energy at which the Greisen-Zatsepin-Kuzmin (GZK) cutoff takes place is given by the threshold for pion photo-production in the collisions of protons and CMB-photons. For an average CMB photon $\left(10^{-3} \mathrm{eV}\right)$, one obtains 


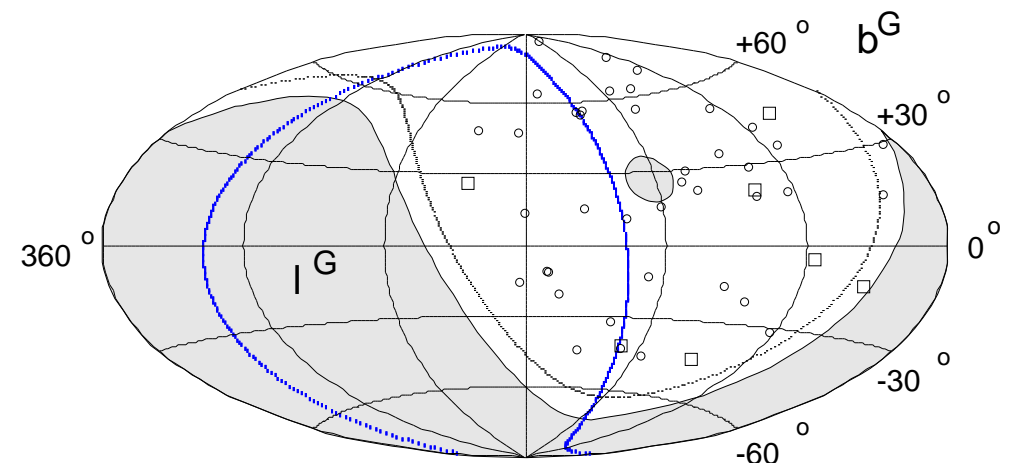

FIGURE 3. Arrival directions (galactic coordinates) of cosmic rays with $E>40 \mathrm{EeV}$, AGASA.

$E_{t h}=7 \times 10^{19} \mathrm{eV}$. The interaction length can be estimated from the pion photoproduction cross section and the $\mathrm{CMB}$ density : $L=(\sigma \rho)^{-1} \simeq 6 \mathrm{Mpc}$.

A recent Monte Carlo [9] calculation, including red shift, pair production and pion photo-production losses, is shown on Figure 4. The loss length $x_{\text {loss }}$ is defined as $x_{l o s s}=\frac{E}{d E / d x}$. Above $100 \mathrm{EeV}$ photo-production processes are dominant and the loss length falls below $13 \mathrm{Mpc}$.

For nuclei, the situation is usually worse. They photo-disintegrate in the CMB and infrared radiations losing on average 3 to 4 nucleons per Mpc when their energy exceeds $2 \times 10^{19} \mathrm{eV}$ to $2 \times 10^{20} \mathrm{eV}$ depending on the IR background density value.

Top-Down production mechanisms predict that, at the source, photons and neutrinos dominate over ordinary hadrons by about a factor four to ten $[5,11]$. An observed dominance of gammas or neutrinos in the supra-GZK range would then be an inescapable signature of a super-heavy particle decay or TD interaction. High energy photons traveling through the Universe produce $e^{+} e^{-}$pairs when colliding with the Infra-Red/Optical (IR/O), CMB or Universal Radio Background (URB) photons. As can be seen on Figure 5 the attenuation length gets below $10 \mathrm{Mpc}$ for photon energies between $3 \times 10^{13} \mathrm{eV}$ and $10^{20} \mathrm{eV}$. In this energy range the Universe is opaque to photons on cosmological scales.

Once the photon has converted, the $e^{+} e^{-}$pair will in turn produce photons mostly via Inverse Compton Scattering (ICS). Those two dominant processes are responsible for the production of electromagnetic (EM) cascades. On Figure 5 one sees that, at the pair production threshold on the CMB photons $\left(2 \times 10^{14} \mathrm{eV}\right)$, conversion occurs on distances of about $10 \mathrm{kpc}$ (a thousand times smaller than for protons at GZK energies) while subsequent ICS of electrons on the CMB in the Thomson regime will occur on even smaller scales $(1 \mathrm{kpc})$.

As a consequence, most photons of ultra high energy will produce, through successive collisions on the various photon backgrounds (URB, CMB, IR/O), lower and lower energy cascades and pile up in the form of a diffuse photon background below $10^{12} \mathrm{eV}$ with a typical power law spectrum of index $\alpha=1.5$. This is a very important fact as measurements of the diffuse gamma ray background in the $10^{7}$ - 

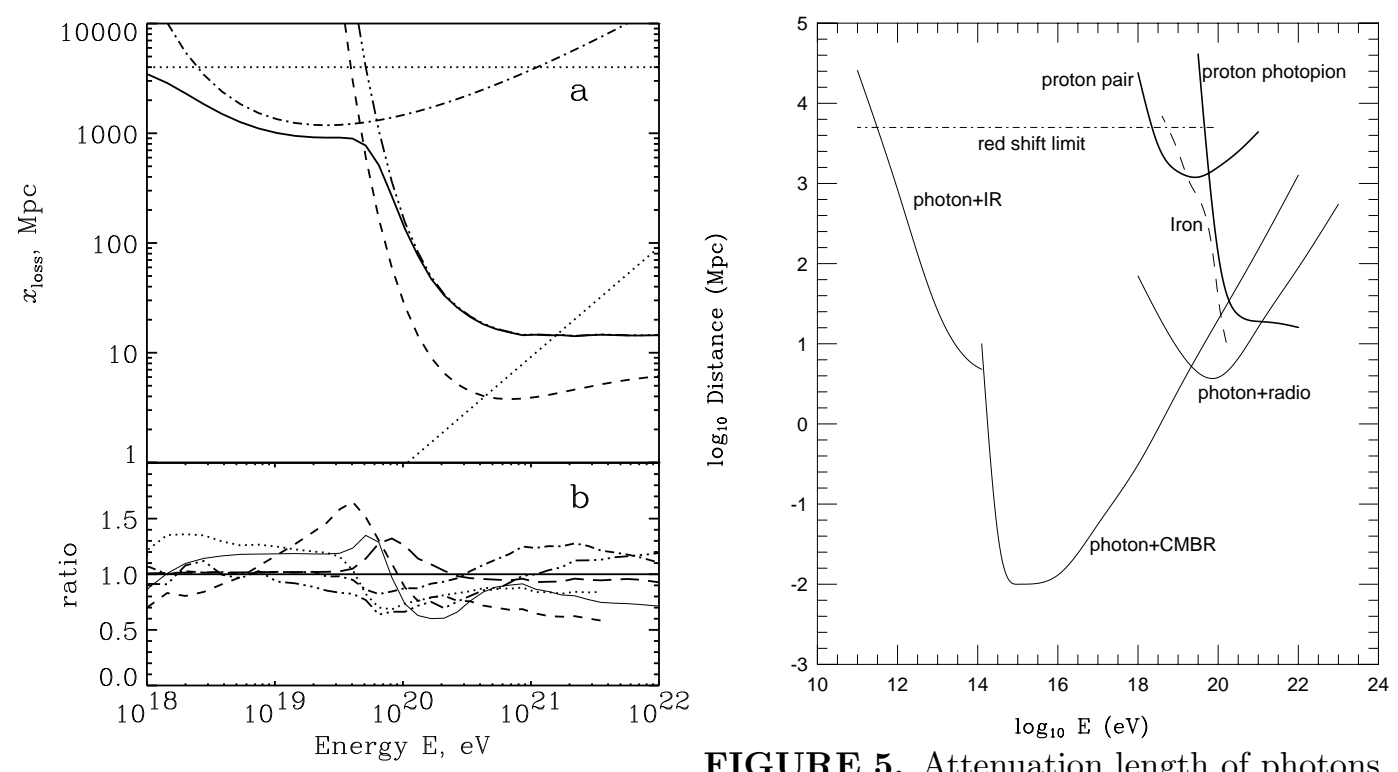

FIGURE 5. Attenuation length of photons,

FIGURE 4. a) Loss length of a proton from protons and iron as a function of energy. Doua recent complete monte calo simulation by ble pair production (not shown) limits the T. Stanev et al. (astro-ph/0003484).

b) Ratios with other calculations. photon attenuation length to about $100 \mathrm{Mpc}$ above $10^{22} \mathrm{eV}$.

$10^{11} \mathrm{eV}$ range done for example by EGRET [12] will impose limits on the photon production fluxes of Top-Down mechanisms and consequently on the abundance of topological defects or relic super-heavy particles.

Neutrinos are the only known particle that can travel through space unaffected even on large distances, carrying intact the properties of the source to the observer. They may prove to be an unambiguous signature of the new physics underlying the production mechanisms.

\section{B Bottom-Up acceleration}

In the conventional acceleration scenarios one distinguishes two types of mechanisms :

- Direct acceleration by very high electric fields in or near very compact objects. This does not naturally provide a power-law spectrum.

- Diffusive shock acceleration in all systems where shock waves are present. This statistical acceleration, known as the Fermi mechanism, naturally provides a power law spectrum.

Hillas has shown [13] that irrespective of the details of the acceleration mechanisms, the maximum energy of a particle of charge $Z e$ within a given site of size $R$ is: 


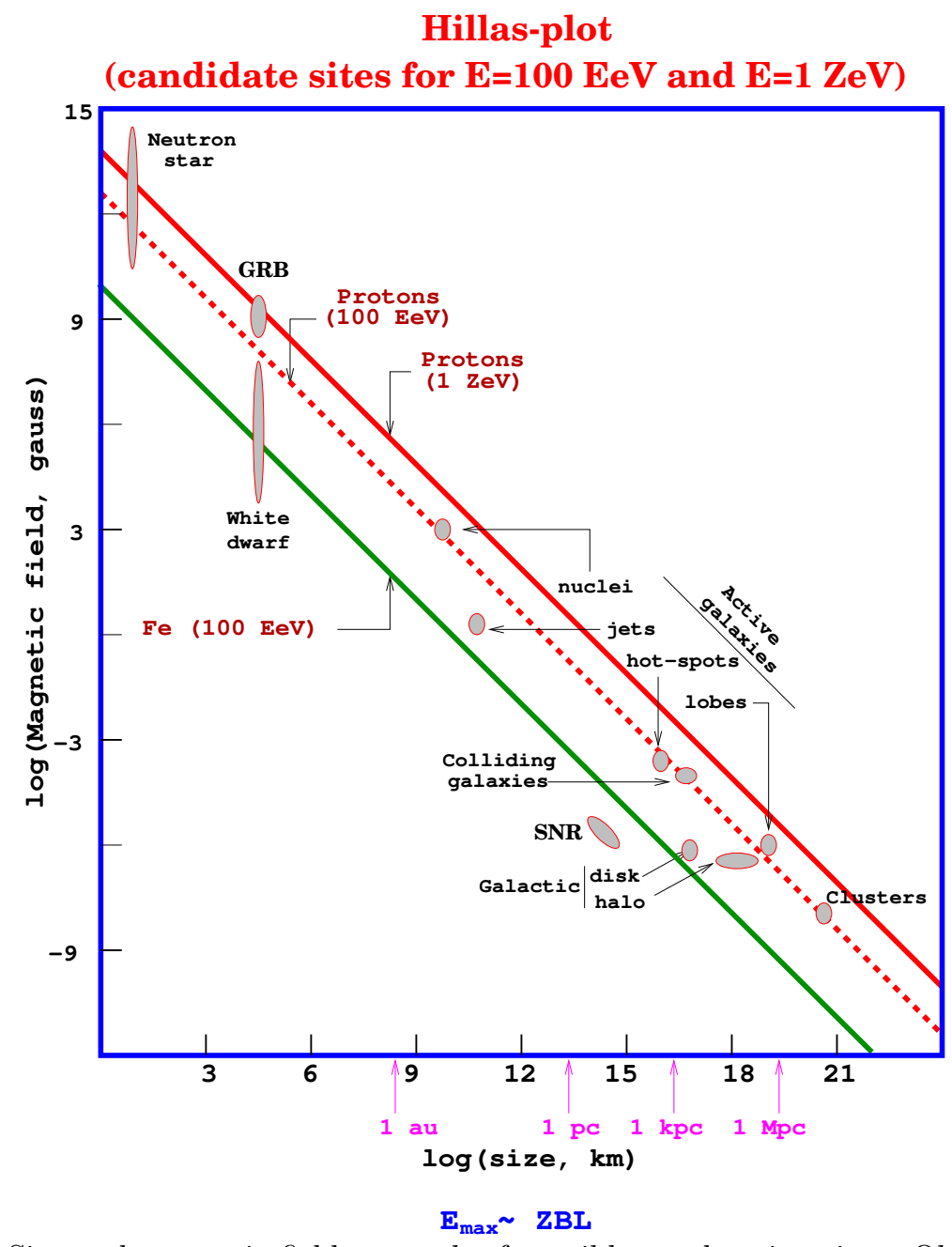

FIGURE 6. Size and magnetic field strength of possible acceleration sites. Objects below the diagonal lines cannot accelerate the corresponding elements above $10^{20} \mathrm{eV}$ or $10^{21} \mathrm{eV}$.

$$
E_{\max } \approx \beta Z\left(\frac{B}{1 \mu \mathrm{G}}\right)\left(\frac{R}{1 \mathrm{kpc}}\right) 10^{18} \mathrm{eV}
$$

where $B$ is the magnetic field inside the acceleration volume and $\beta$ the velocity of the shock wave or the efficiency of the acceleration mechanism. This condition is nicely represented by the Hillas diagram shown in Figure 6. Inspecting this diagram one sees that only a few astrophysical sources satisfy the necessary condition given by Eq. (1).

Let us briefly review them :

Pulsars : From a dimensional analysis, one obtains up to $10^{20}$ volts for the potential drop in a rotating magnetic pulsar. However the high radiation density in the vicinity of the pulsar will produce $e^{+} e^{-}$pairs which short the potential 
drop down to values of about $10^{13} \mathrm{eV}$. A different mechanisms involving Fe nuclei acceleration by relativistic MHD winds has bee put forward [14]. But details of the effectiveness of this mechanism still need to be demonstrated.

AGN cores and jets : Blast waves in AGN jets could in principle lead to a maximum energy of a few tens of EeV [15] and similarly for AGN cores. However those maxima are unlikely to be achieved under realistic conditions due to the interaction of the accelerated protons with very high radiation fields in and around the central engine of an AGN.

FR-II radio galaxies : Radio-loud quasars are characterized by a very powerful central engine ejecting matter along thin extended jets. At the ends of those jets, the so-called hot spots, the relativistic shock wave is believed to be able to accelerate particles up to ZeV energies. FR-II galaxies seem the best potential astrophysical source of UHECR [16]. Unfortunately, no nearby (less than $100 \mathrm{Mpc}$ ) object of this type is visible in the direction of the observed highest energy events.

Gamma Ray Burst : Gamma ray bursters (GRB) are intense sources of gamma rays. The most favored GRB emission model is the "expanding fireball model" where one assumes that a large fireball, as it expands, becomes optically thin hence emitting a sudden burst of gamma rays. The observation of afterglows allowed to measure the red shift of the GRBs hence confirming their cosmological origin. GRB can be shown to accelerate protons up to $10^{20} \mathrm{eV}$ [17]. However in such a framework the UHECR spectrum should clearly show the GZK cut-off.

\section{Top-Down production}

One way to overcome problems related to the acceleration of UHECR and the invisibility of their sources is to introduce a new unstable or meta-stable supermassive $X$-particle. The decay of this $X$-particle produces, among other things, quarks and leptons, resulting in a large cascade of energetic photons, neutrinos and light leptons with a small fraction of protons and neutrons, part of which becomes the UHECR. For this scenario to be observable three conditions must be met:

- The decay must have occurred recently since the decay products must have traveled less than about $100 \mathrm{Mpc}$ because of the attenuation processes discussed above.

- The mass of this new particle must be well above the observed highest energy (100 EeV range), a hypothesis well satisfied by Grand Unification Theories (GUT) whose scale is around $10^{24}-10^{25} \mathrm{eV}$.

- The ratio of the volume density of this particle to its decay time must be compatible with the observed flux of UHECR.

The $X$-particles may be produced by way of two distinct mechanisms:

- Radiation, interaction or collapse of Topological Defects (TD), producing $X$ particles that decay instantly. In those models the TD are leftovers from the 
GUT symmetry-breaking phase transition in the very early universe. Quantitative predictions of the TD density that survives a possible inflationary phase rely on a large number of theoretical hypotheses. Therefore they cannot be taken at face value, although the experimental observation of large differences could certainly be interpreted as the signature of new effects.

- Super-massive meta-stable relic particles from some primordial quantum field, produced after the now commonly accepted inflationary stage of our Universe. However the ratio of their lifetime to the age of the universe requires a fine tuning with their relative abundance. It is worth noting that in some of those scenarios the relic particles may also act as non-thermal Dark Matter.

In all conceivable Top-Down scenarios, photons and neutrinos dominate at the end of the hadronic cascade. This is the important distinction from the conventional acceleration mechanisms.

\section{THE AUGER DETECTOR}

Large area ground based detectors do not observe the incident cosmic ray directly but the Extensive Air Shower (EAS), a very large cascade of particles, they generate in the atmosphere. All experiments aim to measure, as accurately as possible, the direction of the primary cosmic ray, its energy and its nature. There are two major techniques used. One is to build a ground array of sensors spread over a large area, to sample the EAS particle densities on the ground. The other consists in studying the longitudinal development of the EAS by detecting the fluorescence light emitted by the Nitrogen molecules which are excited by the EAS secondaries.

The Auger Observatories ${ }^{2}$ combine both techniques. The full design [10] recommends to instrument two sites, one in the southern hemisphere (Argentina) now under contruction, and one in the north (Utah, USA) to be started in 2003 if approved. A complete sky coverage is a mandatory condition to be able to perform sensible analysis of the possible anisotropies of the UHECR arrival directions. The surface of each site, $3000 \mathrm{~km}^{2}$, will provide statistics of nearly 100 events per year above $100 \mathrm{EeV}$ and the detector is designed to be fully efficient for showers above $10 \mathrm{EeV}$, with a duty-cycle of $100 \%$. The total aperture is $14000 \mathrm{~km}^{2} \mathrm{sr}$ for both sites.

Each station of the ground array is a cylindrical Cherenkov tank of $10 \mathrm{~m}^{2}$ surface and $1.2 \mathrm{~m}$ height filled with filtered water. Three phototubes convert the Cherenkov light into electric signals which are digitized by means of flash ADCs running at $40 \mathrm{MHz}$. Digitisation is triggered each time the Cherenkov ligth signal is equivalent to nearly 4 vertical muons. With a spacing of $1.5 \mathrm{~km}$ between the stations, a $10^{19} \mathrm{eV}$ vertical shower triggers on average 6 of them, which is enough to fully reconstruct the EAS.

2) Named after the French physicist Pierre Auger 


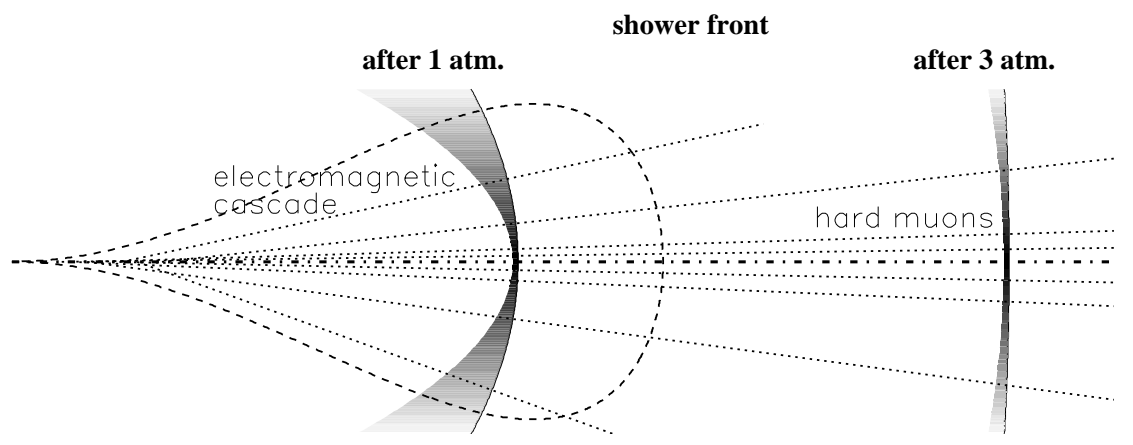

FIGURE 7. Horizontal shower development.

Because of the size of the array, the stations have to work in a stand-alone mode : they are powered by solar panels and batteries and timing is provided by the GPS satellites. Communication with the central buildings, where the central trigger and the data collection take place, is done through a wireless LAN.

The fluorescence telescopes use photo-tubes with a field of view of $1.5^{\circ}$. Each telescope sees an angle of about $30 \times 30$ degrees. On the southern site, three eyes (7 telescopes each) will be installed at the periphery of the array and one (12 telescopes) in the middle, in order for the whole array to be visible by at least one of the telescopes. In hybrid mode ( $10 \%$ of the events) where both the ground array and the fluorescence telescopes monitor the sky, the detector is expected to have on average an energy resolution of $10 \%$ and an angular precision better than $0.3^{\circ}$.

\section{NEUTRINOS}

Although both neutrinos and photons dominate the particle fluxes in Top-Down models, only neutrinos are perfect probes of the UHECR sources. Undeflected by magnetic fields and with very long absorption length they allow to disantangle the characteristics for the sources from the propagation effects. High energy neutrinos can also be produced in Bottom-Up scenarios as secondaries of hadronic interaction. If AGNs, Radio Galaxy lobes or GRBs are UHECR sources they should produce a substantial flux of neutrinos. Finally, along their path in the universe, hadrons will also produce neutrinos via the pion photo-production processes, the so called GZK neutrinos.

The first study on the detection of UHE neutrinos with the Auger detector were done by $[19,20]$. The UHE neutrinos may be detected and distinguished from ordinary hadrons by the shape of the horizontal EAS they produce. At large 
angles, above $60^{\circ}$, hadronic showers have their electromagnetic part extinguished (they have gone through a few equivalent vertical atmosphere $\left(2\right.$ at $60^{\circ}, 3$ at $70^{\circ}, 6$ at $80^{\circ}$ ) and only high energy muons survive. Therefore the front of horizontal hadronic showers is very flat (the radius of curvature is larger than $100 \mathrm{~km}$ ), and very narrow (the time spread is less than $50 \mathrm{~ns}$ ). Unlike hadrons, neutrinos may interact deeply in the atmosphere and can initiate a shower above the detector. This shower will appear as a "normal" one - although horizontal -, with a curved front (radius of curvature of a few $\mathrm{km}$ ), a large electromagnetic component, and a wide signal (spread over a few microseconds) [see Figure 7]. With such important differences and if the fluxes are high enough, neutrinos can be detected and identified.

Bottom-up or top-down processes hardly produce any $\nu_{\tau}$. In the second case one expects of course that at the beginning of the decay chain there is a full equivalence among all flavors, but this symetry breaks down at the end of the fragmentation process where numerous pions are produced, yielding most of the expected neutrino flux.

However, in the event of $\nu_{\mu} \longrightarrow \nu_{\tau}$ oscillations with full mixing - a hypothesis that seems to be suported by the atmospheric neutrino data and the K2K experiment [21]-, one expects, for a very wide range in $\delta m^{2}$, to have as many $\nu_{\mu}$ as $\nu_{\tau}$ in the cosmic ray fluxes. Unlike electrons which do not escape from the rocks or muons that do not produce any visible signal in the atmosphere ${ }^{3}$ the taus, produced in the mountains or in the ground around the Auger array can escape even from deep inside the rock and produce a clear signal if they decay above the detector.

The geometrical configuration that must be met to produce a visible signal is rather severe. Neutrinos must be almost perfectly horizontal (within about $2^{\circ}$ ) and only a few percent of the solid angle is available. They should also interact within the decay range of the tau which must in turn have a reasonable chance of decaying over the size of the detector. The neutrino energy should therefore lie within about $2 \times 10^{17} \mathrm{eV}$ (large flux, $\gamma c \tau \sim 10 \mathrm{~km}$ ) and $6 \times 10^{18} \mathrm{eV}$ (larger average signal, $\gamma c \tau \sim 300 \mathrm{~km}$ ). For downward going $\nu_{\tau}$ most of the observable signals come from interactions taking place in the Andes about $50 \mathrm{~km}$ west of the array, for upward going $\nu_{\tau}$ the interactions occur in the ground all around the array. Our preliminay simulations [22] show that the maximum of the acceptance is reached for neutrino energies of a few $10^{17} \mathrm{eV}$ and that $80 \%$ of the detected neutrinos are upgoing. Figure 8 shows a simulation of the ground trace of a tau, produced by a $3 \times 10^{17} \mathrm{eV}$ neutrino decaying above the ground, as sampled by the Auger stations. The signal is clearly visible and 10 stations pass the 4 vertical muon equivalent trigger requirement (thick circles).

The sensitivity limit in flux of the Auger detector - defined as the neutrino flux giving at least 1 observed event every three years - is shown on Figure 9 together with the expected fluxes from a model calculation by Protheroe [18]. Both limits from atmospheric neutrino interaction [20] and from $\nu_{\tau}$ interactions in the rocks [22]

3) The electro-magnetic halo that surrounds very high energy muons does not spread enough in space to produce a detectable signal in an array of detectors separated by $1.5 \mathrm{~km}$. 


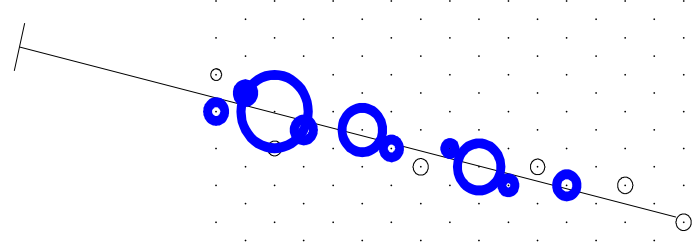

FIGURE 8. Simulation of the ground trace left by a $\tau$ decay shower as produced by a $5 \times 10^{17} \mathrm{eV}$ tau neutrino. Each thick circle represents a triggered station with a surface proportional to the Cherenkov signal. The $\tau$ shower had an energy of $3.63 \times 10^{17} \mathrm{eV}$ and decayed 390 meters above the ground.

are shown. For the sensitivity to tau induced showers (as indicated by the arrows), the tau flux was assumed to be half of the various neutrino fluxes (full mixing hypothesis) presented on the plot. Only the strongest trigger requirement (4 vertical muon equivalent) was simulated.

For standard neutrino interactions in the atmosphere, each site of the Auger observatory reaches $10 \mathrm{~km}^{3}$ water equivalent (w.e.) of target mass, and only the models classified as speculative by Protheroe are expected to yield a detectable signal. However, for tau induced shower the target mass is increased by a factor of nearly 100 and reaches $1000 \mathrm{~km}^{3}$ w.e. at $3 \times 10^{18} \mathrm{eV}$ allowing for a detectable signal even for the lowest expected fluxes. Our preliminary calculation shows that we can expect of the order of 3 to 6 events per year from the GZK neutrinos a very low but almost certain flux.

Only the Auger observatories can perform an optimal detection of these events. Above $10^{17} \mathrm{eV}$ the earth is not transparent to neutrinos and for tau or muon neutrinos successive charged current interactions will degrade the energy below $10^{16} \mathrm{eV}$ mixing the high energy signal with the more standard and far more numerous low energy one. Therefore a maximum of a few $100 \mathrm{~km}$ of rocks should intersect the neutrino trajectory to limit the number of interactions and allow a high energy lepton (above $10^{17} \mathrm{eV}$ ) to escape. Only nearly horizontal neutrinos interacting in mountains or in the top few kilometers of the Earth will allow this.

At $5 \times 10^{18} \mathrm{eV}$ and above, the tau decay length goes beyond $250 \mathrm{~km}$ and the probability of a decay over $50 \mathrm{~km}$ becomes small. Therefore, only the energy range $10^{17}-5 \times 10^{18} \mathrm{eV}$ is available for detection in detectors of about $50 \mathrm{~km}$ in size. At these relatively "low" energies the fluorescence signal is rather small and cannot be seen from far away, therefore the acceptance of a detector like HiRes [1] becomes too small. For the Auger ground array however, the situation is more favorable because 


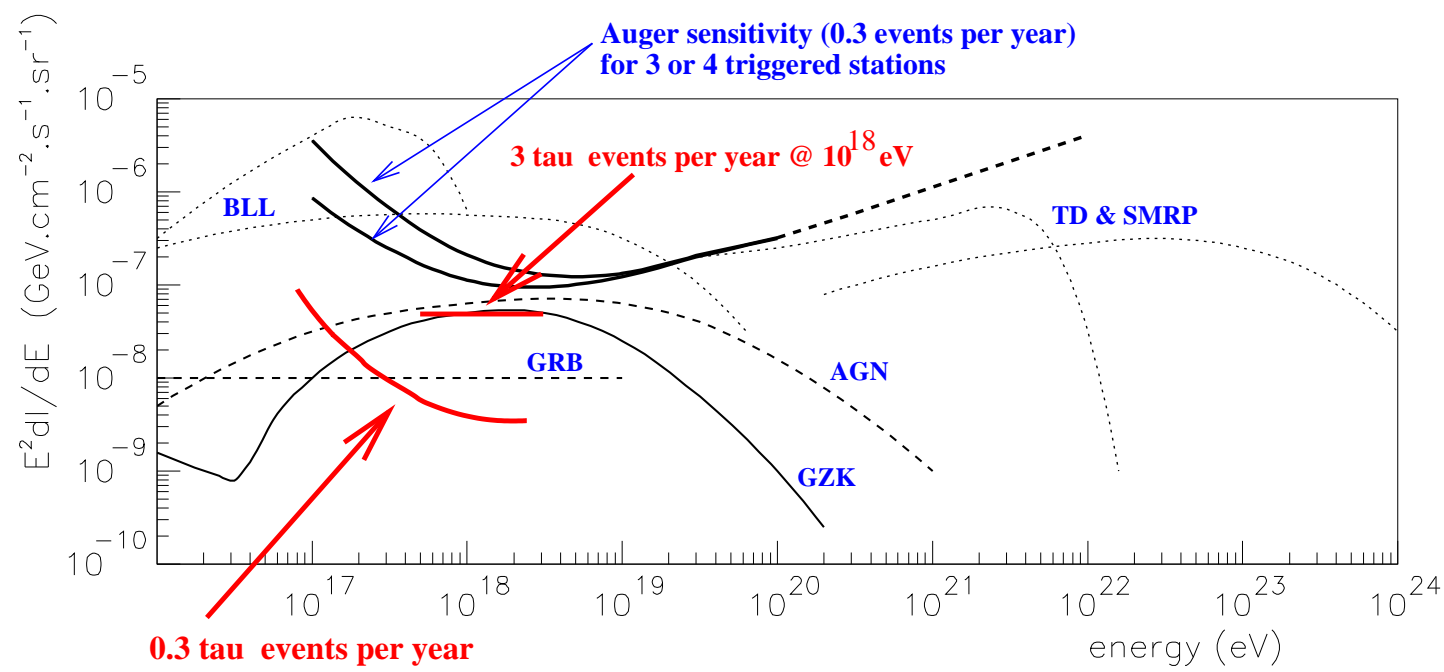

FIGURE 9. Neutrino fluxes and ranking from various sources, dotted lines speculative, dashed probable, solid certain. The two top thick solid lines represent the Auger sensitivity (0.3 event per year) for $\nu_{e}$ and $\nu_{\mu}$ interactions in the atmosphere under two local trigger conditions. Sensitivity to tau induced showers are indicated by the arrows, calculation have been performed only up to neutrino energies of $3 \times 10^{18} \mathrm{eV}$.

the shower spatial size is still large $(10 \mathrm{~km}$ in length and a couple of kilometers in diameter) and because horizontal muons produce a larger signal (by a factor 3 ) when they cross the Cherenkov tanks.

For very large detectors, like EUSO [23], the satellite space fluorescence detector, the energy threshold is above $5 \times 10^{19} \mathrm{eV}$ and the decay probability of the tau over $250 \mathrm{~km}$ is below $10 \%$. Since the flux is 100 times smaller than at $10^{18} \mathrm{eV}$ and since the fiducial mass which is only atmosphere is still of the order of $1000 \mathrm{~km}^{3}$ w.e. the detection of those events from space seems very unlikely.

More work needs to be done to demonstrate that we can properly reconstruct the geometry of those very horizontal showers, zenith angle and altitude above the detector, and therefore correctly estimate their energy. This is the subject of a paper curently in preparation [22].

\section{CONCLUSIONS}

The origin of the highest energy cosmic ray is still not understood. The composition, the shape of the energy spectrum and the distribution of arrivals directions of UHECR will prove to be powerful tools to distinguish between the different production scenarios. 
If UHECR are hadrons accelerated by Bottom-Up mechanisms, they should point back to their sources if magneic field bending is not too largre over $50 \mathrm{Mpc}$, and a visible counterparts is expected. They will have a specific distribution in the sky and a spectrum clearly showing the GZK cutoff.

For Top-Down mechanisms and above $100 \mathrm{EeV}$, one should observe a flux of photons and neutrinos as the photon absorption length increases (up to a few 100 $\mathrm{Mpc}$ ). Below $100 \mathrm{EeV}$ the spectrum shape and composition will depend on the characteristic distance between TD interactions or relic particle decays and Earth, the proton attenuation length and the photon absorption length.

In all cases, and in the hypothesis of full $\nu_{\mu} \rightarrow \nu_{\tau}$ oscillations, tau neutrinos should be visible at a rate which will clearly identify the UHECR production mechanisms, high rate for top down production and low rate for bottom up acceleration. The detection of such a signal -but also its unexpected absence- will allow to fully constrain our models on the origin of the highest energy cosmic rays and shed some light on this mystery. The detection surface and geographic characteristics of the Auger observatories make them optimal detectors for such a discovery.

\section{REFERENCES}

1. J.N. Matthews, C.C.H. Jui, Nucl. Phys. Proc. Suppl. B87, (2000) 411.

2. M. Takeda et al., Astrophys.J. 522, (1999) 225; N. Hayashida et al., astro-ph/0008102.

3. K. Greisen, Phys. Rev. Lett. 16 (1966)748.; G.T.Zatsepin, V.A.Kuzmin, JETP Lett. 4 (1966) 78.

4. S. Yoshida, H. Dai, J.Phys. G24 (1998) 905.

5. P. Bhattacharjee, G. Sigl,Phys.Rept. 327 (2000) 109.

6. X. Bertou, M. Boratav, A. Letessier-Selvon, Int.J.Mod.Phys. A15 (2000) 2181.

7. S. Swordy, private communication. Data points from : LEAP, Proton, Akeno, AGASA, Fly's Eye, Haverah Park, Yakutsk.

8. N. Hayashida et al., astro-ph/9906056; see also Y.Uchihori et al., Astropart.Phys. 13 (2000) 151

9. T. Stanev et al., astro-ph/0003484.

10. The Pierre Auger Project Design Report, Fermilab (1995), www . auger.org/admin/.

11. S. Sarkar, astro-ph/0005256.

12. A. Karle et al, Phys. Rev. Lett. B347 (1995) 161.

13. A. M. Hillas, Annual Review Astron. Astrophys. 22 (1984) 425.

14. P. Blasi, R. I. Epstein, A. V. Olinto, astro-ph/9912240.

15. F. Halzen, E. Zas, Ap. J. 488 (1997) 669.

16. P. L. Biermann, Phys. Rev. D51 (1995) 3450.

17. E. Waxman, Nucl. Phys. Proc. Suppl. 87 (2000) 345

18. R.J. Protheroe, astro-ph/9809144

19. G. Parente, E. Zas, astro-ph/9606091; K. S. Capelle, J. W. Cronin, G. Parente, E. Zas, Astropart. Phys. 8 (1998) 321; S. Coutu, X. Bertou, P. Billoir, John Hopkins Workshop (Neutrinos in the Next Millenium), 1999 (sub. to World Scienc.).

20. P. Billoir, 8th International Workshop on Neutrino Telescopes, (1999) 111; 
21. S. Fukuda et al., astro-ph/0009001 and references therein.

22. X. Bertou, P. Billoir and A. Letessier-Selvon, in preparation.

23. L. Scarsi et al., proposal for the ESA F2/F3 flexible mission, L. Scarsi editor. 